\title{
Metal-enhanced fluorescence of colloidal nanocrystals with nanoscale control
}

\author{
P. P. POMPA*, L. MARTIRADONNA, A. DELLA TORRE, F. DELLA SALA, L. MANNA, M. DE VITTORIO, \\ F. CALABI, R. CINGOLANI AND R. RINALDI \\ National Nanotechnology Laboratory of CNR-INFM, IIT Research Unit, ISUFI, University of Lecce, Via per Arnesano, 73100 Lecce, Italy \\ *e-mail: piero.pompa@unile.it
}

Engineering the spectral properties of fluorophores, such as the enhancement of luminescence intensity, can be achieved through coupling with surface plasmons in metallic nanostructures ${ }^{1-11}$. This process, referred to as metal-enhanced fluorescence, offers promise for a range of applications, including LEDs, sensor technology, microarrays and single-molecule studies. It becomes even more appealing when applied to colloidal semiconductor nanocrystals, which exhibit size-dependent optical properties, have high photochemical stability, and are characterized by broad excitation spectra and narrow emission bands ${ }^{12}$. Other approaches have relied upon the coupling of fluorophores (typically organic dyes) to random distributions of metallic nanoparticles or nanoscale roughness in metallic films $s^{1-4,6,8}$. Here, we develop a new strategy based on the highly reproducible fabrication of ordered arrays of gold nanostructures coupled to $\mathrm{CdSe} / \mathrm{ZnS}$ nanocrystals dispersed in a polymer blend. We demonstrate the possibility of obtaining precise control and a high spatial selectivity of the fluorescence enhancement process.

We fabricated, by electron beam lithography (EBL), highly ordered gold nanopatterns on planar substrates with different shapes and dimensions. A representative example of the twodimensional periodic arrays (200-nm-wide gold nanotriangles, with a period of $400 \mathrm{~nm}$ ) is shown in Fig. 1. A uniform blend of $\mathrm{CdSe} / \mathrm{ZnS}$ nanocrystals, dispersed in a polymer matrix (polymethylmethacrylate, PMMA), was then deposited on the substrates by spin-coating. Depending on several experimental parameters (such as material, shape and dimensions of the metallic nanostructures, as well as the average fluorophore-nanostructure distance $)^{1,2}$, the proximity effect may elicit an efficient coupling between the surface plasmon (SP) resonance band of the metallic nanostructures and the excitation/emission bands of the nanocrystals. Upon proper optimization, such a procedure increases the excitation and/or emission rates of the fluorophores, thus resulting in a strong enhancement of their fluorescence.

The use of a polymeric matrix allowed us to tune the average fluorophore-metallic nanostructure distance, and to obtain a uniform dispersion of the nanocrystals (NCs) onto the substrates (even for large areas), while maintaining good control of the concentration of the active material in the blend. In this study, the optimal thickness of the polymeric blend (in terms of maximum enhancement of NC fluorescence, along with the achievement of a
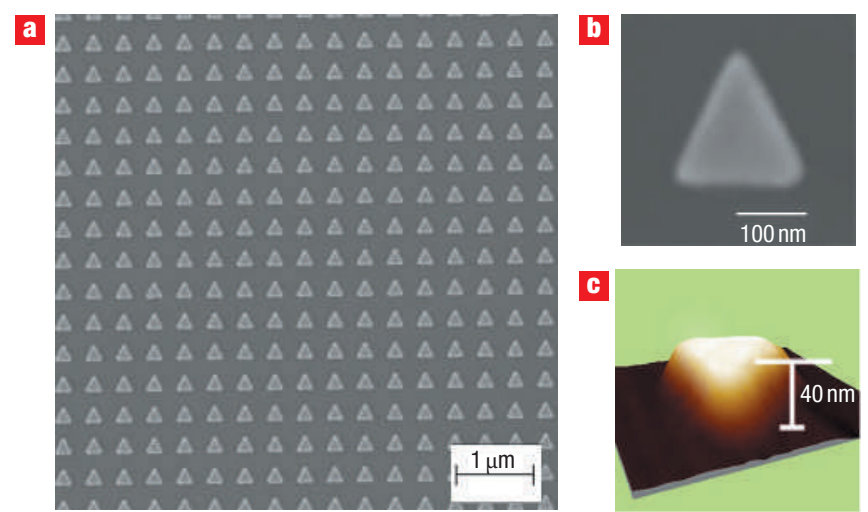

Figure $1 \mathrm{~A}$ typical example of a highly regular gold nanopattern realized by EBL. a, SEM image of a triangular gold array (lateral dimensions: $200 \mathrm{~nm}$; period: $400 \mathrm{~nm}$ ). b, High-resolution SEM image of a single nanotriangle. c, AFM image of the single nanostructure; the height of the triangle was $40 \mathrm{~nm}(5 \mathrm{~nm}$ $\mathrm{Cr}, 35 \mathrm{~nm} \mathrm{Au}$ ), and the mean surface roughness was $1.3 \mathrm{~nm}$.

uniform film; see also Methods) was determined to be $\sim 35 \mathrm{~nm}$, as measured from the surface of the metallic patterns.

Figure 2a shows the fluorescence map of the NC/PMMA blend film deposited on a $100 \times 100 \mu \mathrm{m}^{2}$ metallic nanopattern (200-nm-wide gold nanotriangles with a $400 \mathrm{~nm}$ period; see also Fig. 1). A strong enhancement of the NC fluorescence, induced by metal-enhanced fluorescence (MEF) coupling with the underlying gold nanostructures, can be observed. After normalization over the spatial duty cycle of the pattern, we estimated a fluorescence increase as high as $\sim 30$-fold (Fig. $2 b$ ). The emission spectra of the NCs collected both on $\mathrm{SiO}_{2}$ and on the metallic nanopattern were found to be identical (emission peak, $\lambda_{\max }=580 \mathrm{~nm}$; full-width at half-maximum $(\mathrm{FWHM})=40 \mathrm{~nm}$; see also inset of Fig. $2 \mathrm{~b}$, in which the two normalized spectra are reported), indicating the absence of detectable MEF-induced emission shifts. On the other hand, preliminary experiments performed with NCs emitting at different wavelengths (both in the red and in the blue regions) 

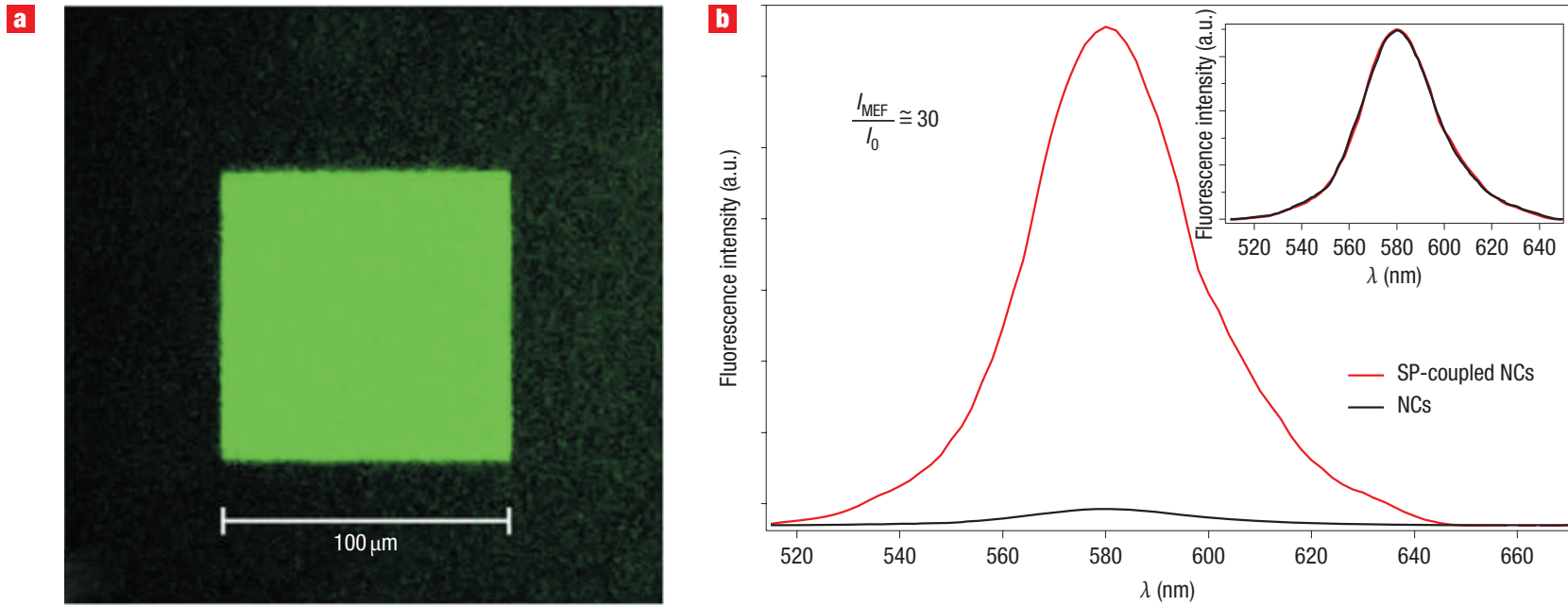

Figure 2 Fluorescence enhancement of colloidal NCs induced by MEF. a, Photoluminescence map of an NC/PMMA blend film deposited on a triangular gold nanopattern (triangle lateral dimensions: $200 \mathrm{~nm}$ ), collected by a confocal microscope. The bright region corresponds to the area in which the luminescence of the NCs is strongly enhanced, due to the coupling with the underlying gold nanoarray. b. Fluorescence spectra of the NCs dispersed in the polymer matrix collected both on $\mathrm{SiO}_{2}$ and on the metallic nanopattern; as shown, a $\sim 30$-fold fluorescence increase was observed. The inset shows normalized fluorescence spectra of the NCs with and without SP coupling; it is evident that the two spectra are identical and maintain the original spectral features of the reference samples in solution $\left(\lambda_{\max }=580 \mathrm{~nm} ; \mathrm{FWHM}=40 \mathrm{~nm}\right)$.

revealed a significant shift of the enhanced fluorescence spectra along with reduced enhancement factors (data not shown). The high enhancement effect observed on the metallic nanopatterns is due to the fluorophore dipole interacting with free electrons in the metal ${ }^{1,2}$, and is marginally affected by the reflection of the emitted photons (this contribution was always less than $2-3 \%$ as compared to the MEF luminescence enhancement). Identical results in terms of fluorescence increase were observed (upon normalization over the corresponding periods) for different patterns in which the spacing between the 200 -nm-wide triangles was varied in the $100-200 \mathrm{~nm}$ range, suggesting a minor role of interparticle interactions in the observed MEF process. The optimal height of the gold nanostructures, which led to maximum fluorescence enhancement of the NCs (namely, a 30-fold increase), was experimentally determined to be in the $30-35 \mathrm{~nm}$ range, in qualitative agreement with theoretical predictions (see in the following).

The potential of this MEF-based approach with colloidal NCs was further investigated by exploiting different gold nanostructures. We fabricated $100-$ and $200-\mathrm{nm}$-wide triangular and cylindrical nanopatterns (with height $35 \mathrm{~nm}$ ), and the same deposition procedure of the NC/PMMA blend was carried out. The results obtained with the four different metallic arrays are reported in Table 1. A remarkable increase in the NC fluorescence emission was observed in all the experiments, although the enhancement factors depended on the specific morphology of the pattern. Also in these experiments, we observed no significant variations in the line-shape of the enhanced NC emission spectra. The highest fluorescence increase was obtained with the 100-nmwide triangular nanoprisms (a 33-fold increase), whereas the NCs coupling with the cylindrical nanopatterns seemed to be less effective.

Theoretical analyses were carried out in order to investigate the spectral features of the SPs of the gold nanostructures used in the MEF experiments. We computed the absorption spectra of gold triangular prisms and cylinders with real dimensions using the Discrete dipole approximation (DDA) ${ }^{13,14}$. The DDA method solves Maxwell's equations by replacing the particle with a cubic array of dipoles, each having an oscillating polarization that is determined by the local dielectric constant, the incoming electromagnetic field, and the interaction with other dipoles. The absorption cross-section can then be obtained from the optical theorem ${ }^{15}$. The DDA is well suited for the computation of the optical properties of particles with arbitrary shapes ${ }^{16-18}$

As shown in Fig. 3, the SP resonance bands of the 100-nmwide gold triangular nanoprisms are characterized by a remarkable overlap with the excitation/emission spectra of the nanocrystals, strongly supporting the high efficiency of MEF coupling observed in our experiments ${ }^{1,2}$. Moreover, a comparison of the absorption efficiencies (see Methods) of the different gold nanostructures (inset of Fig. 3) predicts a trend in the fluorescence enhancement factors that is similar to the one determined from confocal measurements on our patterns (Table 1). Notably, for both shapes, the decrease in the lateral dimensions from 200 to $100 \mathrm{~nm}$ leads to an increase in the MEF efficiency, the best performances being achieved with the 100-nmwide nanoprisms. However, according to the calculated spectra, the $100-\mathrm{nm}$ cylinders are likely to provide a higher enhancement

Table 1 Experimental enhancement factors of NC fluorescence emission (SP-coupled NCs to NCs emission ratio) observed with different nanopatterns: triangular prisms and cylinders with 100- and 200-nm lateral dimensions (the height of these gold nanostructures was $35 \mathrm{~nm}$ ). The reported enhancement factors were normalized over the correspondent spatial duty cycles.

\begin{tabular}{ll}
\hline Gold nanostructure & $\begin{array}{l}\text { Fluorescence } \\
\text { enhancement } \\
\text { factor }\end{array}$ \\
\hline Triangular prisms, $100 \mathrm{~nm}$ & 33 \\
Triangular prisms, $200 \mathrm{~nm}$ & 30 \\
Cylinders, $100 \mathrm{~nm}$ & 26 \\
Cylinders, $200 \mathrm{~nm}$ & 21 \\
\hline
\end{tabular}




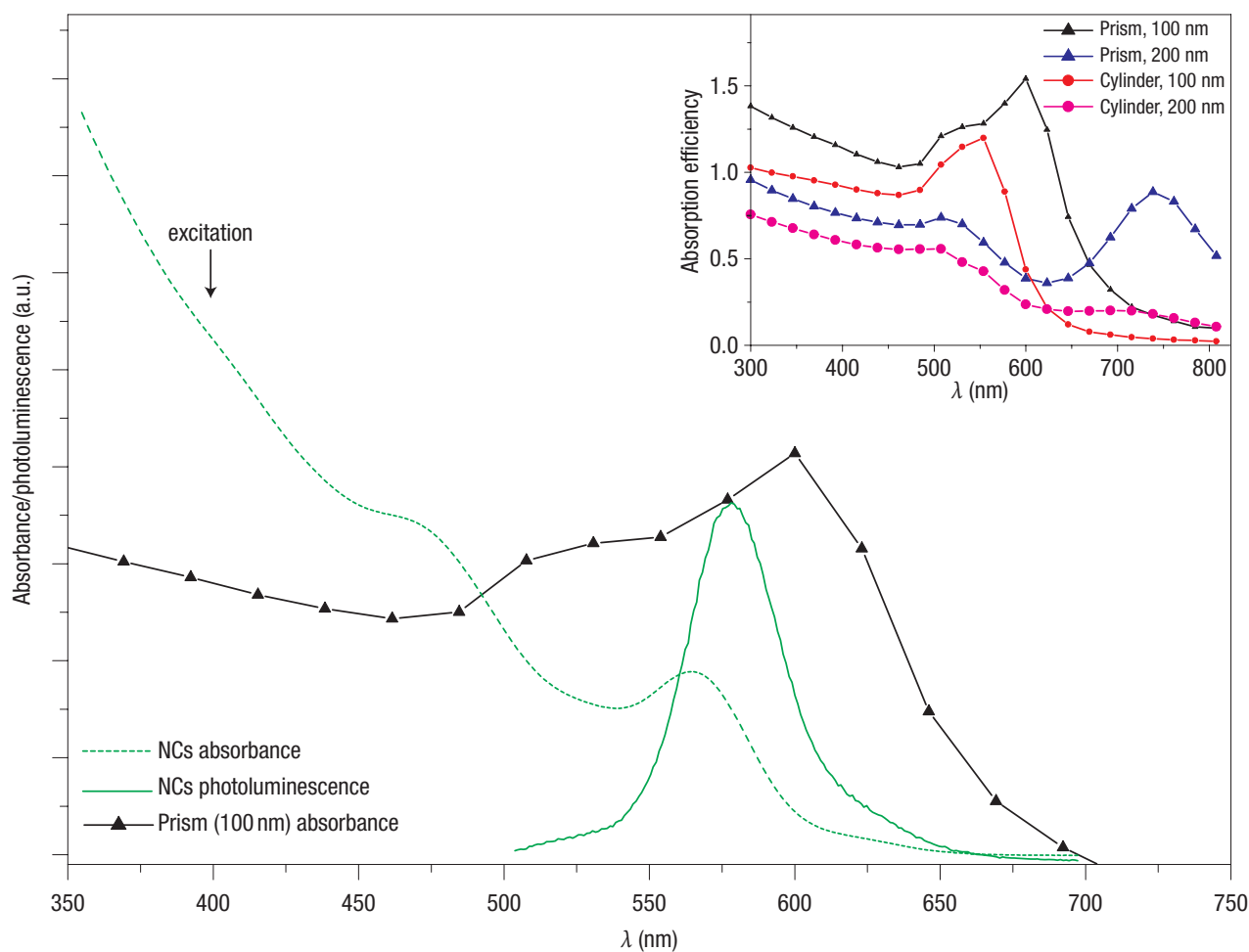

Figure 3 Comparison of the calculated absorption spectrum of triangular gold nanoprisms with the absorption and emission spectra of the colloidal NCs used in the MEF experiments. The lateral dimensions are $100 \mathrm{~nm}$, the height $35 \mathrm{~nm}$. A strong overlap between the NC absorption/emission bands and the SP resonance bands of the gold nanostructure can be observed. The spectral position of the excitation wavelength used in the MEF experiments (405 nm) is indicated. The inset shows the calculated absorption efficiencies of the different gold nanostructures (35 nm height) used in the MEF experiments (for computational details, see Methods).

compared with the 200-nm triangles. This latter discrepancy with the experimental data might be due to slight variations in the geometry of the fabricated gold nanostructures, and/or to the strongly inhomogeneous electric-field distribution within the prisms, which affects the coupling mechanism; indeed, the electric-field enhancement at specific points may be different from the absorption spectra ${ }^{18}$.

In addition, we studied the dependence of the calculated absorption spectra on the height of the gold nanostructures (not shown). We found that the SP resonance band red-shifts significantly as the thickness decreases below $20 \mathrm{~nm}$, thus reducing considerably the spectral overlap with the NCs. The optimized height $(30-35 \mathrm{~nm})$ determined experimentally is therefore consistent with the above analysis.

A key point of the EBL-based MEF strategy (together with the highly controlled and uniform dispersion of NCs onto the substrates) is the possibility to localize the enhancement process with high spatial control, thus opening up attractive perspectives towards a tailored nanoscale design of ultrabright fluorescence emission. This is demonstrated in Fig. 4, where the fluorescence maps of the NC/PMMA blend film deposited on various metallic nanopatterns are displayed. The 200-nm-wide gold nanotriangles form a two-dimensional periodic array (period $=400 \mathrm{~nm}$; see also Fig. 4a) and were designed in order to reproduce accurately, on the micrometre scale, the National Nanotechnology Laboratory acronym. A strong enhancement of NC fluorescence, along with a precise localization of the phenomenon (due to the underlying pattern), was observed (Fig. 4b). In line with the previous results, we found a fluorescence increase as high as $\sim 30$-fold. Importantly, the localization of the enhancement process can be driven down to the nanoscale, as revealed by Fig. 4c-f. Bright MEF signals can be detected in few-micrometresized stripes and even in submicrometre-wide stripes (the 600-nmwide patterns, formed by only two gold nanotriangle columns).

It is worth noting that the proposed approach has several interesting properties: (1) intense multicolour (or even white) fluorescence emission can be obtained by using NCs of different sizes; (2) coupling with the SPs generally induces an increase of the photostability of the active layer ${ }^{1,2}$; 3 ) both the shape and size of the metallic nanopatterns can be accurately designed, at the nanoscale (as shown for instance in Fig. 4), depending on the specific application to be pursued; and (4) single metallic nanostructures can be properly engineered/optimized owing to the nanometre resolution of the EBL. This allows accurate tailoring of SPs over a wide range of wavelengths, and may enable very strong enhancements (theoretically, up to several hundredor thousand-fold increases, even though significant SP losses can be expected in some spectral regions), while preserving a very precise spatial control. Finally, (5) this method can be applied to a wide range of emitters (likely allowing the investigation of the optical properties of new nanomaterials with low emission efficiency). We have demonstrated, for instance, that the identical strategy can be exploited to increase the fluorescence emission of semiconductor quantum rods ${ }^{19}$ (not shown). Moreover, as EBLbased nanopositioning of NCs dispersed in PMMA has recently been demonstrated in our laboratory ${ }^{20}$, we are currently working on the exact localization of single/few emitters (even with different fluorescence properties) on the metallic nanopattern. This may open up attractive perspectives for both research and applicative purposes. 

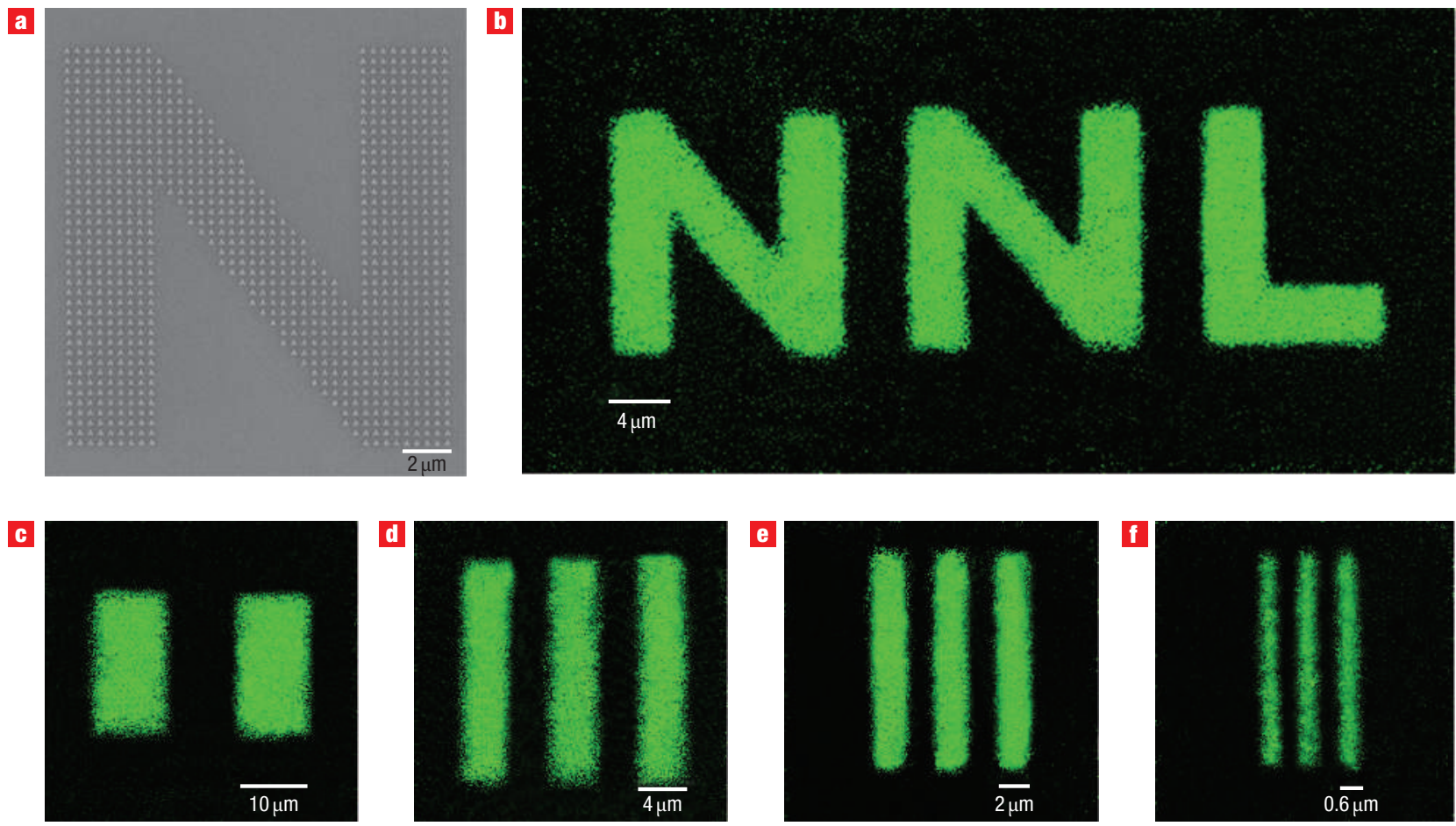

Figure 4 Confocal microscopy images of the NC/PMMA blend film deposited on various metallic nanopatterns. Analogous to Fig. 2, the regions of bright emission are due to the underlying gold nanopatterns. a, SEM image of the ' $N$ ' pattern realized by EBL (200-nm-wide nanotriangles). b, Metallic nanotriangles were defined by EBL in order to form the acronym of our laboratory. c-f, The lateral dimensions of the arrays of nanotriangles were varied from $10 \mu \mathrm{m}$ to only $600 \mathrm{~nm}$, demonstrating the possibility of obtaining very precise control and high spatial selectivity (down to the nanoscale) of the enhancement process.

\section{METHODS}

Highly regular nanopatterns with different shapes and dimensions (100200-nm-wide triangular prisms and cylinders) were designed by EBL (Leica LION LV1) on a positive resist (PMMA-950K, All Resist) spin-coated on a $\mathrm{SiO}_{2}$ substrate. After the development of the resist (120 s at room temperature in a methyl isobutylketone/isopropyl alcohol solution), we deposited, by electron beam evaporation, $5 \mathrm{~nm}$ and $35 \mathrm{~nm}$ of $\mathrm{Cr}$ and $\mathrm{Au}$, respectively $(\mathrm{Cr}$ was necessary to improve gold adhesion onto the $\mathrm{SiO}_{2}$ ), followed by lift-off.

The morphology of the fabricated patterns was assessed by scanning electron microscopy (SEM) and atomic force microscopy (AFM) (AFM images were acquired in the tapping mode).

$\mathrm{CdSe} / \mathrm{ZnS}$ core/shell colloidal NCs were synthesized following traditional methods ${ }^{21,22}$, dispersed in chlorobenzene, and characterized by absorption and photoluminescence spectroscopy (emission peak: $\lambda_{\max }=580 \mathrm{~nm}$; FWHM $=40 \mathrm{~nm}$ ). The NCs were subsequently dispersed in PMMA-950K (final concentrations in chlorobenzene: $\mathrm{C}_{\mathrm{NCs}}=7 \times 10^{-6} \mathrm{M}$ and $\left.\mathrm{C}_{\mathrm{PMMA}}=1.9 \times 10^{-5} \mathrm{M}\right)$ and deposited onto the substrate by spin-coating. The samples were then baked at $180^{\circ} \mathrm{C}$ for $120 \mathrm{~s}$ and characterized by AFM measurements in order to probe the film uniformity and thickness.

Spatially resolved photoluminescence measurements on the samples were performed by confocal microscopy (excitation wavelength, $\lambda_{\text {exc }}=405 \mathrm{~nm}$ ).

In our MEF experiments, we probed different thicknesses of the polymeric blend in the range $20-120 \mathrm{~nm}$ (from the surface of the metallic patterns). We found that maximum enhancement of NC fluorescence was obtained with a 35-nm-thick layer (in this case the layer was very uniform), and we observed a decrease of MEF enhancement as the polymer thickness was increased. In the case of very thin layers $(<35 \mathrm{~nm})$, spatially resolved fluorescence measurements revealed nonuniform distributions of the NCs onto the substrates, so a reliable quantification of the MEF effect was not possible. However, we are currently working on a further optimization of the deposition process, which is likely to allow us to obtain homogeneous layers with a thickness down to few nanometres.
Theoretical calculations have been made using a modified version of the DDSCAT program ${ }^{23}$. The bulk complex dielectric constant for gold has been taken from the tabulated values of Johnson and $\mathrm{Christy}^{24}$. The number of dipoles included in the DDA calculations are 14,464 (for the $100 \mathrm{~nm}$ triangular prisms), 57,904 (for the $200 \mathrm{~nm}$ triangular prisms), 26,368 (for the $100 \mathrm{~nm}$ cylinders) and 105,168 (for the $200 \mathrm{~nm}$ cylinders). The dimensionless absorption efficiency used is defined as the absorption cross-section, normalized to the area $A$ of the particle, as seen from the direction of the incident radiation; in the case of a prism (cylinder), $A$ is the area of the triangle (circle).

The theoretical results are related to the absorption efficiency of gold particles in vacuum, whereas in the experiments they are actually embedded in a PMMA matrix and deposited on a $\mathrm{SiO}_{2}$ substrate (via a 5-nm thin layer of $\mathrm{Cr}$, see above). The different refractive index of the surrounding environment and the coupling to the substrate might cause red-shifts of the plasmon peak $^{25,26}$, which are difficult to reproduce quantitatively using the DDA method ${ }^{18}$

Received 19 May 2006; accepted 26 September 2006; published 3 November 2006.

\section{References}

1. Lakowicz, J. R. Radiative decay engineering: biophysical and biomedical applications. Anal. Biochem. 298, 1-24 (2001).

2. Aslan, K. et al. Metal-enhanced fluorescence: an emerging tool in biotechnology. Curr. Opin. Biotechnol. 16, 55-62 (2005).

3. Neal, T. D., Okamoto, K. \& Scherer, A. Surface plasmon enhanced emission from dye doped polymer layers. Opt. Express 13, 5522-5527 (2005).

4. Shimizu, K. T., Woo, W. K., Fisher, B. R., Eisler, H. J. \& Bawendi, M. G. Surface-enhanced emission from single semiconductor nanocrystals. Phys. Rev. Lett. 89, 117401 (2002).

5. Tovmachenko, O. G., Graf, C., van den Heuvel, D. J., van Blaaderen, A. \& Gerritsen, H. C. Fluorescence enhancement by metal-core/silica-shell nanoparticles. Adv. Mater. 18 91-95 (2006).

6. Aslan, K., Lakowicz, J. R. \& Geddes, C. D. Rapid deposition of triangular silver nanoplates on planar surfaces: application to metal-enhanced fluorescence. J. Phys. Chem. B 109, 6247-6251 (2005)

7. Farahani, J. N., Pohl, D. W., Eisler, H.-J. \& Hecht, B. Single quantum dot coupled to a scanning optical antenna: a tunable superemitter. Phys. Rev. Lett. 95, 017402 (2005). 
8. Nakamura, T. \& Hayashi, S. Enhancement of dye fluorescence by gold nanoparticles: analysis of particle size dependence. Jpn J. Appl. Phys. 44, 6833-6837 (2005).

9. Huang, F. M., Festy, F. \& Richards, D. Tip-enhanced fluorescence imaging of quantum dots. Appl. Phys. Lett. 87, 183101 (2005).

10. Zhang, J., Ye, Y.-H., Wang, X., Rochon, P. \& Xiao, M. Coupling between semiconductor quantum dots and two-dimensional surface plasmons. Phys. Rev. B 72, 201306 (2005).

11. Brolo, A. G. et al. Surface plasmon-quantum dot coupling from arrays of nanoholes. J. Phys. Chem. B 110, 8307-8313 (2006).

12. Klimov, V. I. Semiconductor and Metal Nanocrystals (Marcel Dekker, New York, 2004).

13. Purcell, E. M. \& Pennypacker, C. R. Scattering and absorption of light by nonspherical dielectric grains. Astrophys. J. 186, 705-714 (1973).

14. Draine, B. T. \& Flatau, P. J. Discrete-dipole approximation for scattering calculations. J. Opt. Soc Am. A 11, 1491-1499 (1994).

15. Boren, C. F., Huffman, D. R. Absorption and Scattering of Light by Small Particles (Wiley-Interscience, New York, 1983).

16. Yang, W.-H., Schatz, G. C. \& Van Duyne, R. P. Discrete dipole approximation for calculating extinction and Raman intensities for small particles with arbitrary shapes. J. Chem. Phys. 103, 869-875 (1995).

17. Sosa, I. O., Noguez, C. and Barrera, R. G. Optical properties of metal nanoparticles with arbitrary shapes. J. Phys. Chem. B 107, 6269-6275 (2003).

18. Kelly, K. L., Coronado, E., Zhao, L. L. \& Schatz, G. C. The optical properties of metal nanoparticles: The influence of size, shape, and dielectric environment. J. Phys. Chem. B 107, 668-677 (2003).

19. Manna, L., Scher, E. C. \& Alivisatos, A. P. Synthesis of soluble and processable rod-, arrow-, teardrop-, and tetrapod-shaped CdSe nanocrystals. J. Am. Chem. Soc. 122, 12700-12706 (2000).

20. Martiradonna, L., Stomeo, T., De Giorgi, M., Cingolani, R. \& De Vittorio, M. Nanopatterning of colloidal nanocrystals emitters dispersed in a PMMA matrix by e-beam lithography. Microelectron. Eng. 83, 1478-1481 (2006)
21. Dabbousi, B. O. et al. (CdSe)ZnS Core-shell quantum dots: Synthesis and characterization of a size series of highly luminescent nanocrystallites. J. Phys. Chem. B 101, $9463-9475$ (1997).

22. Reiss, P., Bleuse, J. \& Pron, A. Highly luminescent CdSe/ZnSe core/shell nanocrystals of low size dispersion. Nano Lett. 2, 781-784 (2002).

23. Draine, B. T. \& Flatau, P. J. DDSCAT 6.1 (University of California at San Diego, California, 2004) 24. Johnson, P. B. \& Christy, R. W. Optical constants of the noble metals. Phys. Rev. B 6, 4370-4379 (1972).

25. Jensen, T. R. et al. Nanosphere lithography: Effect of the external dielectric medium on the surface plasmon resonance spectrum of a periodic array of silver nanoparticles. J. Phys. Chem. B 103, 9846-9853 (1999)

26. Malinsky, M. D., Kelly, K. L., Schatza, G. C. \& Van Duyne, R. P. Nanosphere lithography: Effect of substrate on the localized surface plasmon resonance spectrum of silver nanoparticles. J. Phys. Chem. B 105, 2343-2350 (2001).

Acknowledgements

The authors gratefully acknowledge help from L. Carbone and L. L. del Mercato in the synthesis of NCs and for AFM characterizations, and the expert technical assistance of E. D'Amone and G. Epifani. This work was partially supported by the Italian Ministry of Research through MIUR "FIRB" project (RBLA03ER38_001). P.P.P. and L.M. would also like to thank the AAs association for fruitful discussions.

Competing financial interests

The authors declare that they have no competing financial interests.

Reprints and permission information is available online at http://npg.nature.com/reprintsandpermissions/ 